\title{
Integrable Hierarchy of Higher Nonlinear Schrödinger Type Equations
}

\author{
Anjan KUNDU
}

Saha Institute of Nuclear Physics, Theory Group \& Centre for Applied Mathematics

\& Computational Science, 1/AF Bidhan Nagar, Calcutta 700 064, India

E-mail: anjan.kundu@saha.ac.in

URL: http://www.saha.ac.in/theory/anjan.kundu/

Received August 14, 2006, in final form October 17, 2006; Published online November 10, 2006

Original article is available at http://www.emis.de/journals/SIGMA/2006/Paper078/

\begin{abstract}
Addition of higher nonlinear terms to the well known integrable nonlinear Schrödinger (NLS) equations, keeping the same linear dispersion (LD) usually makes the system nonintegrable. We present a systematic method through a novel Eckhaus-Kundu hierarchy, which can generate higher nonlinearities in the NLS and derivative NLS equations preserving their integrability. Moreover, similar nonlinear integrable extensions can be made again in a hierarchical way for each of the equations in the known integrable NLS and derivative NLS hierarchies with higher order LD, without changing their LD.
\end{abstract}

Key words: NLSE \& DNLSE; higher nonlinearity; linear dispersion preservation; integrable Eckhaus-Kundu hierarchy

2000 Mathematics Subject Classification: 35G20; 37C85; 35G25; 37E99

\section{Introduction}

Nonlinear Schrödinger equation (NLSE):

$$
i q_{t}+q_{x x}-2 \sigma|q|^{2} q=0
$$

as well as derivative NLSE (DNLSE)

$$
i q_{t}+q_{x x}-i \alpha\left(|q|^{2} q\right)_{x}=0
$$

arising in different physical context [1], represent well known integrable systems. The integrability of such systems as well as the stability of soliton solutions inherent to such equations are believed to be due to a fine balance between their linear dispersive and nonlinear collapsing terms. Therefore, in some physical situations, which demand addition of higher nonlinear terms to $(1.1)$ or $(1.2)[2,3,4,5,6,7]$, this balance apparently is lost and the system turns into a nonintegrable one, not allowing analytic solutions $[8,9,10]$. On the other hand, together with the well known NLSE and DNLSE (1.1)-(1.2) there exists a tower of equations in their integrable hierarchies corresponding to higher conserved charges, where increasingly higher nonlinear terms do arise. However at the same time higher order linear dispersive terms also appear in these integrable equations, apparently to compensate for the higher nonlinearities and for restoring the balance. For example, the next equation in the NLSE hierarchy (evolving with time $t_{3}$ ) has the form

$$
q_{t_{3}}+q_{x x x}+6 \sigma^{2}|q|^{2} q_{x}=0
$$

with higher nonlinearity $|q|^{2} q_{x}$, which however has a compensating higher order linear dispersive term: $q_{x x x}$. 
Nevertheless, in physical systems quite often one needs to add higher nonlinear terms (including nonlinear derivative terms) without increasing the linear dispersion (LD) of the system. An excellent example of such a system is the Johnson's equation [2], which was derived to solve an important hydrodynamic problem for analyzing Stokes instability in fluid flow near the critical value of $k h$. This equation which is uniformly valid for any $k h$ is given in the form:

$$
A_{t}-a_{1} A_{x x}-a_{2}|A|^{2} A+a_{3}|A|^{4} A+i a_{4}|A|^{2} A_{x}-i a_{5}\left(|A|^{2}\right)_{x} A-a_{6} \theta_{t} A=0,
$$

where $a_{i}$ are real numbers and $\theta_{x}=\delta|A|^{2}$. Evidently this physical system contains the same linear dispersive (LD) term $a_{1} A_{x x}$ as in the NLSE and DNLSE together with their associated nonlinear terms, whereas it also has additional 5-th order nonlinearity along with nonlinear terms containing derivatives (described by the terms with coefficients $a_{3}, a_{4}$ as well as $a_{6}$ ). As shown in [2], at criticality: $k h=1.363$ and all $a_{i}$ have known values with $a_{2}=0$.

Another example of such a physical system is the Benney's hydrodynamic equation [3] describing long and short wave interaction. Therefore it is natural to ask whether one can add higher nonlinearity to the original integrable NLS and DNLS equations (1.1)-(1.2) without changing their LD, and at the same time preserving their integrability. Moreover, we may enquire whether it is possible to do this in a systematic and hierarchical way.

We focus on these intriguing questions and find that there exists an integrable hierarchy of Eckhaus-Kundu equations [11, 12], extending the NLS and DNLS equations to higher nonlinearities and thus it provides a conclusive answer to the above posed questions. Moreover, such a hierarchy with the addition of nonlinear terms in a recursive way without changing their LD and the integrability can also be constructed for each of the equations with higher order LD in the known integrable NLSE and DNLSE hierarchies.

\section{Higher nonlinear NLSE and DNLSE}

It has been shown [11] that under a nonlinear transformation of the field $q \rightarrow Q=q e^{-i \theta}$ with arbitrary gauge function $\theta$ the NLSE yields an integrable higher nonlinear equation

$$
i Q_{t}+Q_{x x}-2 \sigma|Q|^{2} Q-\left(\theta_{t}+\theta_{x}^{2}-i \theta_{x x}\right) Q+2 i \theta_{x} Q_{x}=0 .
$$

The DNLSE is extended similarly under the same field transformation to

$$
i Q_{t}+Q_{x x}-i \alpha\left(|Q|^{2} Q\right)_{x}-\left(\theta_{t}+\theta_{x}^{2}-i \theta_{x x}\right) Q+\theta_{x}\left(2 i Q_{x}+\alpha|Q|^{2} Q\right)=0 .
$$

It is interesting to note that the generation of such new integrable equations can be linked to the gauge transformation of the corresponding Lax equations: $\Phi_{x}=U \Phi, \Phi_{t}=V \Phi$, of the NLSE and DNLSE to the new systems $\tilde{\Phi}_{x}=\tilde{U} \tilde{\Phi}, \tilde{\Phi}_{t}=\tilde{V} \tilde{\Phi}$, with the gauge transformed Lax operators $\tilde{U}=h U h^{-1}+h_{x} h^{-1}$ and $\tilde{V}=h V h^{-1}+h_{t} h^{-1}$, where the gauge matrix $h \in U(1)$ is given by $h=e^{i \theta \sigma_{3}}$, with arbitrary gauge field $\theta(x, t)$. Note that though both equations $(2.1),(2.2)$ have been studied quite extensively $[13,14,15]$, the investigations were confined mostly to the particular functional choice for $\theta$ as $\theta=\delta \int^{x}\left|Q\left(x^{\prime}\right)\right|^{2} d x^{\prime}$, with a real parameter $\delta$. Under this choice the NLSE (1.1) is extended to the Eckhaus-Kundu (EK) equation [11, 12, 16, 17, 18]

$$
i Q_{t}+Q_{x x}-2 \sigma|Q|^{2} Q+\delta^{2}|Q|^{4} Q+2 i \delta\left(|Q|^{2}\right)_{x} Q=0
$$

while the DNLSE (1.2) turns into a similar equation

$$
i Q_{t}+Q_{x x}-i \alpha\left(|Q|^{2} Q\right)_{x}+\frac{\delta}{2}(2 \delta-\alpha)|Q|^{4} Q+2 i \delta\left(|Q|^{2}\right)_{x} Q=0
$$


Note that in both above equations higher nonlinear terms including derivative and 5-th power nonlinearity are added to the NLSE and DNLSE, without changing the linear dispersive term and without spoiling the integrability of the system. It was detected [11] that, for the parameter choice $\alpha=2 \delta$ and $\alpha=\delta,(2.4)$ turns into two other well known equations, e.g. Chen-Lie-Liu [19] and Gerdjikov-Ivanov equations [20].

We intend to generalize now this concept and find the general form for additional nonlinear terms, for both NLSE and DNLSE, such that they can be included without altering the linear dispersion and the integrability. We thus find a novel hierarchy of EK equations which provides a systematic way for such construction. We observe first that in equation (2.1) the gauge function $\theta$ should be chosen through its $x$ and $t$ derivatives as $\theta_{x}=\delta|q|^{2}, \theta_{t}=i \delta\left(q^{*} q_{x}-q q_{x}^{*}\right)$, for obtaining higher nonlinear NLSE (2.3), while one should choose in (2.2), $\theta_{x}=\delta|q|^{2}, \theta_{t}=$ $i \delta\left(q^{*} q_{x}-q q_{x}^{*}\right)+\frac{3}{2} \alpha \delta|q|^{4}$, to get the extended DNLSE (2.4). It is important to note that, in both these cases the necessary condition $\left(\theta_{x}\right)_{t}=\left(\theta_{t}\right)_{x}$ must hold, which follows here from the validity of the NLSE and DNLSE themselves for the field function $q(t, x)$.

Our idea, therefore, is to widen this choice of $\theta$ for finding a hierarchical rule, by using the conservation relation $i \rho_{t}+I_{x}=0$. Such integrable systems, as is well known, possess infinite number of conserved quantities in involution, and we can make a systematic and recursive choice for $\theta_{x}^{(n)}=\rho^{(n)}$ and $\theta_{t}^{(n)}=i I^{(n)}, n=0,1,2, \ldots$ for constructing our hierarchy.

Interestingly, we can apply the idea of this construction to each of the higher equations in the NLSE and DNLSE hierarchies with higher order LD to generate a new integrable EK hierarchy with higher nonlinear terms, but with the same LD.

To remove any possible confusion, we should remark here that the integrable EK hierarchies we discover here for the NLSE and DNLSE, together with the known equations (2.3), (2.4) as well as the well known Chen-Lie-Liu and Gerdjikov-Ivanov equations etc. do not appear in the celebrated classification list of Mikhailov et. al [21, 22], since it excludes equations which are related to the enlisted equations through gauge or invertible nonlinear transformations.

\section{Recursive formulas and general framework for conservation law}

We first present a general framework for deriving the required conservation rule for both NLSE and DNLSE. We start with the linear set of Lax equations: $\Phi_{x}=U \Phi, \Phi_{t}=V \Phi$ with $\Phi=$ $\left(\Phi_{1}, \Phi_{2}\right)$ and eliminate $\Phi_{2}$ from both these equations. That would yield from the first Lax equation a second order linear equation for $\Phi_{1}$ :

$$
\Phi_{1 x x}=\left(\frac{U_{12 x}}{U_{12}}\right) \Phi_{1 x}+\left(U_{11 x}+U_{11}^{2}+U_{12} U_{21}-\frac{U_{12 x}}{U_{12}} U_{11}\right) \Phi_{1},
$$

where $U_{i j}$ are matrix elements of the space-Lax operator $U$. Representing now $\Phi_{1}=e^{U_{11} x+\phi}$ the linear equation (3.1) turns into a Riccati equation for $\phi_{x}=\nu(\lambda)$ :

$$
U_{12}\left(\frac{\nu}{U_{12}}\right)_{x}+2 U_{11} \nu+\nu^{2}=U_{12} U_{21}
$$

which by expanding $\nu(\lambda)$ in powers of $\lambda^{-1}$ generates an infinite set of conserved densities $c_{n}$, $n=0,1,2, \ldots$. On the other hand from the time-Lax equation we derive $\phi_{t}=V_{11}+V_{12} \frac{\Phi_{2}}{\Phi_{1}}$, while the space-Lax equation gives $\frac{\Phi_{2}}{\Phi_{1}}=\frac{\phi_{x}}{U_{12}}$, yielding $\phi_{t}=V_{11}+\frac{V_{12}}{U_{12}} \phi_{x}$. After taking $x$-derivative this gives the crucial relation

$$
\nu_{t}=\left(V_{11}+\frac{V_{12}}{U_{12}} \nu\right)_{x} .
$$


Note that we can derive now the conservation law and hence the hierarchical expressions for the gauge field $\theta$ from (3.3) for concrete systems of NLSE and DNLSE by inserting the corresponding expressions for the Lax matrix elements $U_{i j}, V_{i j}$.

\section{Higher nonlinear hierarchy from NLSE}

We concentrate first on the NLSE system for deriving its integrable hierarchy of higher nonlinear equations with the same linear dispersive term. For NLSE the conservation relations were established explicitly in [23]. Using the known form for the Lax matrices of the NLSE:

$$
\begin{aligned}
& U_{11}=-i \lambda, \quad U_{12}=\sqrt{\sigma} q, \quad U_{21}=\sqrt{\sigma} q^{*} \quad \text { and } \\
& V_{11}=-i\left(2 \lambda^{2}+\sigma|q|^{2}\right), \quad V_{12}=\sqrt{\sigma}\left(2 \lambda q+i q_{x}\right),
\end{aligned}
$$

we derive the corresponding Riccati equation from (3.2) as

$$
2 i \lambda \nu=\nu^{2}-\sigma|q|^{2}+q\left(\frac{\nu}{q}\right)_{x}
$$

Since $\nu$ vanishes at $|\lambda| \rightarrow \infty$, expanding it in spectral parameter $\nu=\sum_{n=0} \frac{c_{n}}{(2 i \lambda)^{n+1}}$, we find a recursion relation from (4.2) for the densities of the conserved quantities:

$$
c_{n+1}=q\left(\frac{c_{n}}{q}\right)_{x}+\sum_{k=0}^{n-1} c_{k} c_{n-k-1}, \quad \text { for } \quad n \geq 1
$$

with $c_{0}=-\sigma|q|^{2}, c_{1}=-\sigma q q_{x}^{*}, c_{2}=-\sigma\left(q q_{x x}^{*}-\sigma|q|^{4}\right)$, etc. This gives a methodical way for evaluating the infinite set of conserved quantities $C_{n}=\int c_{n} d x, n=0,1,2, \ldots$ Since these conserved quantities are related to the NLSE, which is an integrable system in the Liouville sense, all of them must be in involution: $\left\{C_{n}, C_{m}\right\}_{\mathrm{PB}}=0[23,24]$. This may be checked by using the fundamental PB relation $\left\{q(x), q^{*}(y)\right\}_{\mathrm{PB}}=\delta(x-y)$ and the boundary condition $\lim _{x \rightarrow \pm \infty}|q| \rightarrow 0$. We focus now on crucial relation (3.3), which using the relevant Lax matrix elements for the NLSE derives the conservation relation

$$
\nu_{t}=-i\left(\sigma|q|^{2}+\left(2 i \lambda-\frac{q_{x}}{q}\right) \nu\right)_{x}
$$

Expanding further $\nu(\lambda)$ in parameter $\lambda$, we get the hierarchy of relations for the densities of conserved quantities

$$
c_{n t}=i\left(-c_{n+1}+\frac{q_{x}}{q} c_{n}\right)_{x}, \quad n=0,1,2, \ldots,
$$

where all densities $c_{n}$ can be evaluated from the recurrence relation (4.3). Choosing therefore $\rho^{(n)}=-\frac{1}{\sigma} c_{n}$ and $I^{(n)}=\frac{1}{\sigma}\left(c_{n+1}-\frac{q_{x}}{q} c_{n}\right)$ we can derive finally the required conservation law

$$
i \rho_{t}^{(n)}+I_{x}^{(n)}=0, \quad n=0,1,2, \ldots
$$

Considering few starting values $n=0,1, \ldots$ we can easily evaluate their explicit forms as

$$
\rho^{(0)}=|q|^{2}, \quad I^{(0)}=-\left(q q_{x}^{*}-q^{*} q_{x}\right), \quad \rho^{(1)}=q q_{x}^{*}, \quad I^{(1)}=\left(\left|q_{x}\right|^{2}-q q_{x x}^{*}+\sigma|q|^{4}\right),
$$


etc. Therefore we conclude that we can have a series of choices for the gauge function $\theta$ given by

$$
\begin{aligned}
& \theta_{x}^{(n)}=\delta\left[\rho^{(n)}\right]=-\frac{\delta}{\sigma}\left[c_{n}\right], \\
& \theta_{t}^{(n)}=\delta\left[i I^{(n)}\right]=\frac{\delta}{\sigma}\left[i\left(c_{n+1}-\frac{q_{x}}{q} c_{n}\right)\right]=\frac{\delta}{\sigma}\left[i\left(c_{n+1}-\frac{Q_{x}}{Q} c_{n}+i \frac{\delta}{\sigma} c_{n}^{2}\right)\right],
\end{aligned}
$$

for each values of $n=0,1,2, \ldots$, where $[x]=x+x^{*}\left(\right.$ or $\left.i\left(x-x^{*}\right)\right)$ indicates nontrivial real valued combination of $x$ together with convenient normalization by some constants. For example, one should evaluate the term appearing in the above expression as

$$
\left[i \frac{Q_{x}}{Q} c_{n}\right]=i\left(\frac{Q_{x}}{Q} c_{n}-\frac{Q_{x}^{*}}{Q^{*}} c_{n}^{*}\right)
$$

Note that by inserting this choice for $\theta^{(n)}$ in higher order equation (2.1) one can generate a novel hierarchy of EK equations for $n=0,1,2, \ldots$. At the bottom of this hierarchy, as we see from (4.7), lies the simplest equation (2.3). However it is important to note that since the gauge field $\theta$ in our construction should be a real function, when it is chosen through conserved densities $c_{n}$, which in general can be complex valued as evident from (4.3), real combination of its expression must be taken with proper care, along with any convenient normalization. Such a manipulation with $c_{n}$ obviously does not affect the conservation law (4.5), since it is a linear equation in $c_{n}$. For example, for $n=0$ when $c_{0}$ is real we can take $\theta_{x}^{(0)}=\delta|q|^{2}$, however for $n=1$, when $c_{1}$ is complex valued, we must have

$$
\theta_{x}^{(1)}(q)=-\frac{\delta}{\sigma}\left[c_{1}\right]=-i \delta\left(q^{*} q_{x}-q q_{x}^{*}\right)
$$

to make it real. Similarly we get for $n=2$,

$$
\theta_{x}^{(2)}(q)=-\frac{\delta}{\sigma}\left[c_{2}\right]=\delta\left(q^{*} q_{x x}+q q_{x x}^{*}-2 \sigma|q|^{4}\right) .
$$

The simplest and the lowest in the EK hierarchy with $n=0$ is given explicitly by (2.3). We therefore present the details for the next higher nonlinear equation with $n=1$ in the EK hierarchy using (4.7), while the other equations with $n \geq 2$ can be derived in a similar way. Following the above formulation we get for $n=1$ :

$$
\theta_{x}^{(1)}(q)=i \delta T(q), \quad \theta_{t}^{(1)}(q)=-\delta\left(E(q)+2 \sigma|q|^{4}\right),
$$

where

$$
T(q) \equiv\left(q^{*} q_{x}-q q_{x}^{*}\right), \quad E(q) \equiv 2 q_{x}^{*} q_{x}-\left(q q_{x x}^{*}+q^{*} q_{x x}\right) .
$$

The consistency of (4.9) can also be checked independently by using the NLSE for the field $q$. However, since the equation for the transformed field $q \rightarrow Q$ with $q=Q e^{i \theta(Q)}$ is of our interest, we have to rewrite all the above expressions completely in terms of the new field, by expressing the functions $\theta_{x}(q), \theta_{t}(q)$ through $Q$. For this purpose we derive

$$
q^{*} q_{x}=\left(Q^{*} e^{-i \theta(Q)}\right)\left(\left(Q_{x}+i \theta_{x}(Q) Q\right) e^{i \theta(Q)}\right)=\left(Q^{*} Q_{x}\right)+i \theta_{x}(Q)|Q|^{2},
$$

which evaluates

$$
\begin{aligned}
& T(q)=T(Q)+2 i \theta_{x}^{(1)}(Q)|Q|^{2}, \\
& E(q)=E(Q)+4\left(-i \theta_{x}^{(1)}(Q) T(Q)+\theta_{x}^{(1)}(Q)^{2}|Q|^{2}\right),
\end{aligned}
$$


where

$$
T(Q) \equiv\left(Q^{*} Q_{x}-Q Q_{x}^{*}\right), \quad E(Q)=2\left|Q_{x}\right|^{2}-\left(Q^{*} Q_{x x}+Q Q_{x x}^{*}\right) .
$$

Resulting

$$
\theta_{x}^{(1)}(Q)=i \delta\left(Q^{*} Q_{x}-Q Q_{x}^{*}\right)-2 \delta \theta_{x}^{(1)}(Q)|Q|^{2}
$$

which gives

$$
\theta_{x}^{(1)}(Q)=i \frac{\delta}{M(Q)} T(Q), \quad M(Q) \equiv 1+2 \delta|Q|^{2}
$$

and similarly

$$
\theta_{t}^{(1)}(Q)=\delta\left(E(Q)+2 \sigma|Q|^{4}+4\left(-i \theta_{x}^{(1)}(Q) T(Q)+\theta_{x}^{(1)}(Q)^{2}|Q|^{2}\right)\right),
$$

where $T(Q), E(Q)$ are as defined in (4.12).

As a result we derive the next higher nonlinear equation with $n=1$ in the EK hierarchy from the NLSE as

$$
\begin{aligned}
i Q_{t} & +Q_{x x}-2 \sigma|Q|^{2} Q-\delta\left(\left(E(Q)+2 \sigma|Q|^{4}+\frac{1}{M(Q)} T_{x}(Q)\right) Q+2 \frac{1}{M(Q)} T(Q) Q_{x}\right) \\
& +\frac{\delta^{2}}{M(Q)} T(Q)\left(\frac{1}{M(Q)}\left(4 \delta T(Q)+\left(|Q|^{2}\right)_{x}\right)-3 T(Q)\right) Q=0
\end{aligned}
$$

with $E(Q), M(Q), T(Q)$ as defined above. Note that this is an integrable equation with higher nonlinearities, but with the same second order LD as in the NLSE. Note that (4.15) contains an additional coupling constant $\delta$ entering in different powers. Therefore we may simplify this equation by considering $\delta$ to be small and ignoring all terms with higher powers in $\delta$, which yields

$$
i Q_{t}+Q_{x x}-2 \sigma|Q|^{2} Q+2 \delta\left(-\sigma|Q|^{4} Q-Q^{*}\left(Q_{x}\right)^{2}+Q_{x x}^{*} Q^{2}\right)=0 .
$$

However it should be noted that though the simpler equation (4.16) as such is a nonintegrable system, it might be meaningful for physical applications and useful information can be extracted for it from its integrable variant (4.15) through limiting procedure.

The general form of the EK hierarchy can be generated similarly in the same form (2.1) where the series of gauge functions $\theta_{t}^{(n)}, \theta_{x}^{(n)}, n=0,1,2, \ldots$ are chosen as (4.8). This would construct finally a new hierarchy of integrable EK equations emerging from the NLSE in the form

$$
i Q_{t}+Q_{x x}-2 \sigma|Q|^{2} Q+\frac{\delta}{\sigma}\left(\left(\left[i\left(-c_{n+1}+\frac{Q_{x}}{Q} c_{n}\right)\right]-i\left[c_{n x}\right]\right) Q-2 i\left[c_{n}\right] Q_{x}\right)=0
$$

where the real-value sign $[\cdot]$ should be properly evaluated in explicit calculations. The conserved densities $c_{n}=c(Q)_{n}, n=0,1,2$, appearing in equations (4.17) are to be obtained systematically from (4.3) and then expressed consistently in terms of the field $Q$, as we have demonstrated in the case $n=1$. We stress again that the integrable hierarchy of EK equations (4.17), contains higher and higher nonlinearities (including nonlinear dispersive terms), but has the same second order LD as in the original NLSE. Note that the density of the conserved quantities, as evident from (4.3), introduces an additional derivative resulting the appearance of higher nonlinear dispersive terms at each higher step. However this should not be confused with the linear dispersive term $Q_{x x}$, which remains the same for the whole EK hierarchy, which in fact is our aim. 


\subsection{LD preserving EK hierarchy from NLSE hierarchy}

Recall that the well known integrable NLSE hierarchy is generated by the Lax pair $\left(U, V^{(k)}\right)_{\mathrm{NLSE}}$, $k=1,2,3, \ldots$ by the flatness condition $U_{t_{k}}-V_{x}^{(k)}+\left[U, V^{(k)}\right]=0$, with the $k$ th equation having the $k$ th order LD. $k=2$ corresponds to the NLSE (1.1), while $k=3$ yields the integrable equation (1.3). It should be noted also that while the space-Lax operator $U$ is the same as that of the NLSE, the time-Lax operator $V^{(k)}, k=1,2,3, \ldots$ is different for different equations in this hierarchy with higher time $t_{k}$. Nevertheless, it is interesting that, the idea we have developed above for generating nonlinear EK hierarchy from the NLSE preserving its LD, can be successfully implemented to each of the $k$ equations in the known NLSE hierarchy. Moreover, since the Riccati equation (4.2) and consequently the recurrence relation (4.3) yielding the conserved quantities depend only on the Lax operator $U$, they should be the same for all equations in the hierarchy with any $k$. Therefore one can make the same choice (4.8) for the gauge function $\theta_{x}^{(n)}, n=0,1, \ldots$. However since the time evolution (4.4) with respect to higher time $t_{k}$ would depend explicitly on the matrix elements $V_{11}^{(k)}, V_{12}^{(k)}, k=1,2,3, \ldots$ as evident from (3.3), this would be different for different $k$, leading to new choices for the $\theta_{t_{k}}^{(n)}$, obeying the consistency $\left(\theta_{x}^{(n)}\right)_{t_{k}}=\left(\theta_{t_{k}}^{(n)}\right)_{x}$. Thus the $k$ th equation in the NLSE hierarchy (with $k$ th order LD) would generate EK type nonlinear hierarchy with $n=0,1, \ldots$, preserving its linear ( $k$ th order) dispersive term and the integrability.

Since we have presented above in detail the $k=2$ case, i.e the NLSE, we give here in brief the construction of the EK hierarchy for the next equation (1.3) with 3rd-order LD. It is easy to check that (1.3) can be extended through gauge transformation to the hierarchical form:

$$
\begin{aligned}
Q_{t_{3}} & +Q_{x x x}+6 \sigma^{2}|Q|^{2} Q_{x}+3 i \theta_{x}^{(n)} Q_{x x}+3\left(i \theta_{x x}^{(n)}-\left(\theta_{x}^{(n)}\right)^{2}\right) Q_{x} \\
& -\left(-i \theta_{t_{3}}^{(n)}+3 \theta_{x}^{(n)} \theta_{x x}^{(n)}-i \theta_{x x x}^{(n)}+i\left(\theta_{x}^{(n)}\right)^{3}+6 i \sigma^{2} \theta_{x}^{(n)}|Q|^{2}\right) Q=0 .
\end{aligned}
$$

For deriving now $\nu(\lambda)_{t_{3}}$ and consequently $\theta_{t_{3}}^{(n)}$ we have to use, as mentioned above, the relevant elements of the corresponding time-Lax operator $V^{(3)}$ given by [24]:

$$
\begin{aligned}
& V_{11}^{(3)}=4 i \lambda^{3}+\left(2 i \lambda|q|^{2}-\left(q q_{x}^{*}-q^{*} q_{x}\right)\right) \sigma, \\
& V_{12}^{(3)}=\left(-4 \lambda^{2} q+2 i \lambda q_{x}-2 \sigma|q|^{2} q\right) \sqrt{\sigma} .
\end{aligned}
$$

Inserting consistent $\theta_{t_{3}}^{(n)}, \theta_{x}^{(n)}$, in (4.18) we can generate another new EK type integrable higher nonlinear hierarchy for $n=0,1, \ldots$ with the same linear dispersive term $Q_{x x x}$.

We however will not derive here the general form of this hierarchy; instead present the simplest and the lowest equation with $n=0$. We find that the corresponding gauge function $\theta^{(0)}$ in this case can be chosen as

$$
\begin{aligned}
& \theta_{x}^{(0)}=\delta|Q|^{2}, \\
& \theta_{t_{3}}^{(0)}=\delta\left(Q^{*} Q_{x x}+Q Q_{x x}^{*}-Q_{x}^{*} Q_{x}-3 \sigma^{2}|Q|^{4}-3 \delta^{2}|Q|^{6}+3 i \delta|Q|^{2}\left(Q^{*} Q_{x}-Q Q_{x}^{*}\right)\right) .
\end{aligned}
$$

Inserting (4.20) in hierarchy (4.18) we reduce it to its lowest order equation

$$
\begin{aligned}
Q_{t_{3}} & +Q_{x x x}+6 \sigma^{2}|Q|^{2} Q_{x}+\delta\left\{3 i|Q|^{2} Q_{x x}+3\left(i\left(|Q|^{2}\right)_{x}-\delta|Q|^{4}\right) Q_{x}\right. \\
& +\left(i\left(|Q|^{2}\right)_{x x}-\frac{3}{2} \delta\left(|Q|^{4}\right)_{x}\right) Q+i\left(Q^{*} Q_{x x}+Q Q_{x x}^{*}-Q_{x}^{*} Q_{x}+3 \sigma^{2}|Q|^{4}\right. \\
& \left.\left.-4 \delta^{2}|Q|^{6}+3 i \delta|Q|^{2}\left(Q^{*} Q_{x}-Q Q_{x}^{*}\right)\right) Q\right\}=0 .
\end{aligned}
$$

Note that this is an integrable equation with higher nonlinearities up to 7-th power in the field as well as with nonlinear dispersive terms, but having the same LD term $Q_{x x x}$ as in (1.3). 


\section{Higher nonlinear hierarchy from DNLSE}

The steps formulated above may be applied now for finding the nonlinear integrable hierarchy from the DNLSE with the same 2nd-order LD as in the original equation. For this purpose we shall use the general form of the conservation relation presented in (3.2)-(3.3), but customize them for this particular case by considering Lax operators [25] associated with the DNLSE:

$$
\begin{aligned}
& U=-i \frac{\lambda^{2}}{4} \sigma^{3}+\frac{i}{2} \lambda \sqrt{\alpha}\left(\begin{array}{cc}
0 & q^{*} \\
q & 0
\end{array}\right), \\
& V=i\left(\frac{\lambda^{4}}{8}-\frac{\alpha}{4} \lambda^{2}|q|^{2}\right) \sigma^{3}+i\left(\begin{array}{cc}
0 & g^{*} \\
g & 0
\end{array}\right), \quad g=\frac{1}{4} \lambda \sqrt{\alpha}\left(-\lambda^{2} q+2 i q_{x}+2 \alpha|q|^{2} q\right) .
\end{aligned}
$$

Note however that unlike the NLSE the required conservation relations for the DNLSE are not readily available in the literature in explicit form and have to be derived carefully by inserting relevant expressions from (5.1). This gives from (3.1) the linear equation for $\Phi_{1}$ as

$$
\Phi_{1 x x}=\left(\frac{q_{x}^{*}}{q^{*}}\right) \Phi_{1 x}+\left(-\frac{\mu^{2}}{16}-\mu \frac{\alpha}{4}|q|^{2}+i \frac{\mu}{4} \frac{q_{x}^{*}}{q^{*}}\right) \Phi_{1}, \quad \mu \equiv \lambda^{2}
$$

yielding in turn the Riccati equation for the DNLS system:

$$
q^{*}\left(\frac{\nu}{q^{*}}\right)_{x}+\nu^{2}-\frac{i}{2} \mu \nu+\mu \frac{\alpha}{4}|q|^{2}=0 .
$$

Using now the consistent expansion in the spectral parameter: $\nu=i \sum_{n=0} \frac{c_{n}}{\mu^{n}}$, we get the recursion relation

$$
\frac{i}{2} c_{n+1}=q^{*}\left(\frac{c_{n}}{q^{*}}\right)_{x}+i \sum_{k=0}^{n} c_{k} c_{n-k}, \quad \text { for } n \geq 0, \quad c_{0}=-\frac{1}{2} \alpha|q|^{2},
$$

which systematically generates all higher conserved densities starting from

$$
c_{1}=\alpha\left(i q^{*} q_{x}+\frac{1}{2} \alpha|q|^{4}\right), \quad c_{2}=2 \alpha\left(q^{*} q_{x x}-2 i \alpha|q|^{2}\left(q^{*} q_{x}+\frac{1}{4} q q_{x}^{*}\right)-\frac{\alpha^{2}}{2}|q|^{6}\right), \quad \text { etc. }
$$

The infinite set of conserved quantities $C_{n}=\int c_{n} d x, n=0,1, \ldots$ for the DNLSE, which is a completely integrable system, must be in involution. This can be checked directly by using its fundamental PB structure: $\left\{q(x), q^{*}(y)\right\}=\delta_{x}(x-y)$ and the vanishing boundary condition for the field: $|q| \rightarrow 0$, at $x \rightarrow \pm \infty$. Using further the Lax matrix elements (5.1) we derive from (3.3) the crucial conservation law

$$
\nu_{t}=\left(-i \mu \frac{\alpha}{4}|q|^{2}+\left(-\frac{\mu}{2}-i \frac{q_{x}^{*}}{q^{*}}+\alpha|q|^{2}\right) \nu\right)_{x} .
$$

Expanding $\nu(\mu)$ through spectral parameter $\mu$ yields the important relation

$$
c_{n t}=\left(-\frac{1}{2} c_{n+1}+\left(-i \frac{q_{x}^{*}}{q^{*}}+\alpha|q|^{2}\right) c_{n}\right)_{x}, \quad n=0,1, \ldots, \quad c_{0}=-\frac{1}{2} \alpha|q|^{2} .
$$

For deriving therefore the higher nonlinear hierarchy from the DNLSE we can use the general form (2.2) with the choice for the gauge function

$$
\begin{aligned}
\theta_{x}^{(n)}=\delta\left[\rho^{(n)}\right] & =\frac{\delta}{\alpha}\left[c_{n}\right] \\
\theta_{t}^{(n)}=\left[i I^{(n)}\right] & =\frac{\delta}{\alpha}\left[-\frac{1}{2} c_{n+1}+\left(-i \frac{q_{x}^{*}}{q^{*}}+\alpha|q|^{2}\right) c_{n}\right] \\
& =\frac{\delta}{\alpha}\left[-\frac{1}{2} c_{n+1}+\left(-i \frac{Q_{x}^{*}}{Q^{*}}+\alpha|Q|^{2}\right) c_{n}-\frac{\delta}{\alpha} c_{n}^{2}\right], \quad n=0,1,2, \ldots,
\end{aligned}
$$


where the real-value sign [.] has the same meaning as explained above. For example, for $n=0$ with real $c_{0}$ we have $\theta_{x}^{(0)}=\delta|q|^{2}$, while for $n=1$ with complex valued $c_{1}$ we must take

$$
\theta_{x}^{(1)}=\delta\left(i\left(q^{*} q_{x}-q q_{x}^{*}\right)+\alpha|q|^{4}\right), \quad \text { etc. }
$$

with the similar reason holding also for the choice of $\theta_{t}^{(n)}$.

This derives finally the new hierarchy of integrable higher nonlinear equations from the DNLSE as

$$
\begin{aligned}
i Q_{t} & +Q_{x x}-i \alpha\left(|Q|^{2} Q\right)_{x} \\
& -\frac{\delta}{\alpha}\left(\left(\left[-\frac{1}{2} c_{n+1}-i \frac{Q_{x}^{*}}{Q^{*}} c_{n}\right]-i\left[c_{n x}\right]\right) Q-2 i\left[c_{n}\right] Q_{x}\right)=0
\end{aligned}
$$

where again all real-valued expressions should be evaluated carefully and $c_{n}=c(Q)_{n}$ are to be obtained recursively from (5.4), expressed explicitly through transformed field $Q$. Thus the tower of equations (5.8) with $n=0,1,2, \ldots$ would represent a novel EK hierarchy, at the bottom of which with $n=0$ giving

$$
\theta_{x}^{(0)}=\delta|q|^{2}, \quad \theta_{t}^{(0)}=\delta\left(i\left(q^{*} q_{x}-q q_{x}^{*}\right)+\frac{3}{2} \alpha|q|^{4}\right),
$$

lies equation (2.4).

It is worth noting that in analogy with the known reductions [11], the parameter choice $\alpha=2 \delta$ in (5.8) would yield a new Chen-Lie-Liu type hierarchy, while $\alpha=\delta$ would generate another Gerdjikov-Ivanov type hierarchy. All these equations however have the same LD given by $Q_{x x}$.

For demonstrating the novelty of hierarchy (5.8), we take up $n=1$, giving the next new equation with

$$
\theta_{x}^{(1)}(q)=\delta\left(i T(q)+\alpha|q|^{4}\right), \quad \theta_{t}^{(1)}(q)=\delta\left(E(q)+3 i \alpha T(q)|q|^{2}+2 \alpha^{2}|q|^{6}\right),
$$

where

$$
T(q)=q^{*} q_{x}-q q_{x}^{*}, \quad E(q)=2\left|q_{x}\right|^{2}-\left(q^{*} q_{x x}+q q_{x x}^{*}\right) .
$$

For further application, as was performed also in the NLSE case, we have to express the gauge fields $\theta_{x}^{(1)}(q), \theta_{t}^{(1)}(q)$ given by (5.9) in terms of the transformed field $Q$ and rewrite

$$
T(q)=T(Q)+2 i \theta_{x}^{(1)}|Q|^{2} \quad \text { and } \quad E(q)=E(Q)+4\left(-i \theta_{x}^{(1)}(Q) T(Q)+\theta_{x}^{(1)}(Q)^{2}|Q|^{2}\right) .
$$

Note that though the expressions for $\theta_{x}^{(1)}(q)$ and $\theta_{t}^{(1)}(q)$ differ for the cases of NLSE and DNLSE, the entries $T(q)$ and $E(q)$ appearing in them have the same form for both these cases. Using the above result after some algebra we arrive at the required expressions

$$
\begin{aligned}
\theta_{x}^{(1)}(Q)= & \frac{\delta}{M(Q)}\left(i T(Q)+\alpha|Q|^{4}\right), \\
\theta_{t}^{(1)}(Q)= & \delta\left(E(Q)+3 i \alpha T(Q)|Q|^{2}-6 \alpha \theta_{x}^{(1)}(Q)|Q|^{4}+2 \alpha^{2}|Q|^{6}\right. \\
& \left.+4\left(-i \theta_{x}^{(1)}(Q) T(Q)+\theta_{x}^{(1)}(Q)^{2}|Q|^{2}\right)\right),
\end{aligned}
$$

where

$$
\begin{aligned}
& M(Q)=1-2 \delta|Q|^{2}, \quad T(Q)=Q^{*} Q_{x}-Q Q_{x}^{*} \quad \text { and } \\
& E(Q)=2\left|Q_{x}\right|^{2}-\left(Q^{*} Q_{x x}+Q Q_{x x}^{*}\right) .
\end{aligned}
$$


By inserting (5.10) with (5.11) in the general form (2.2) we derive finally for $n=1$ the next higher nonlinear equation in the EK hierarchy linked to the DNLSE as

$$
i Q_{t}+Q_{x x}-i \alpha\left(|Q|^{2} Q\right)_{x}-\left(\theta_{t}^{(1)}+\left(\theta_{x}^{(1)}\right)^{2}-i \theta_{x x}^{(1)}\right) Q+\theta_{x}^{(1)}\left(2 i Q_{x}+\alpha|Q|^{2} Q\right)=0,
$$

where expressions for $\theta_{t}^{(1)}(Q)$ and $\theta_{x}^{(1)}(Q)$ expressed through $T(Q), E(Q)$ as in (5.10)-(5.11), together with $\theta_{x x}^{(1)}(Q)$ by extracting the $x$-derivative, are to be inserted in equation (5.12) to get its explicit higher nonlinear form. As we see this integrable equation contains additional higher nonlinear terms up to 7th power nonlinearity together with many nonlinear dispersive terms, though with the same second-order LD: $Q_{x x}$, as in the original DNLSE.

\subsection{LD preserving EK hierarchy from DNLSE hierarchy}

The integrable hierarchy of the DNLSE is generated similarly to the NLSE by the associated Lax pair $\left(U, V^{(k)}\right)_{\text {DNLSE }}, k=1,2,3, \ldots$. The space-Lax operator $U$ corresponds to the DNLSE (5.1), while the time-Lax operator $V^{(k)}, k=1,2,3, \ldots$ is different for different higher time $t_{k}$, yielding through the flatness condition the $k$ th equation in this hierarchy with $k$ th order LD. $k=2$ corresponds to the DNLSE (1.2), while $k=3$ yields the next integrable equation [25]

$$
q_{t_{3}}-q_{x x x}+3 i \sigma\left(|q|^{2} q_{x}\right)_{x}+\frac{3}{2} \sigma^{2}\left(|q|^{4} q\right)_{x}=0 .
$$

The above scheme we have implemented to the DNLSE (with $k=2$ ) can also be applied to each of the $k>2$ equations for generating integrable EK type hierarchy preserving its $k$ th order LD. Moreover, the Riccati equation (5.3), the determining relation (5.4) for the conserved quantities and consequently the choice for the gauge function $\theta_{x}^{(n)}, n=0,1, \ldots$ as given in (5.7), would be the same for all equations in this hierarchy, since they all depend on the space-Lax operator $U$ only. However the relations like (5.5), (5.6) and consequently the consistent expression for $\theta_{t_{k}}^{(n)}$, which are linked to the time evolution should be calculated using the matrix elements of the time-Lax operator $V^{(k)}, k=1,2,3, \ldots$

Thus we can construct in principle higher nonlinear hierarchy with $n=0,1, \ldots$, from any of the $k$ th equation in the DNLSE hierarchy, preserving its $k$ th order LD and the integrability. In practice however each case with higher $k$ would be more complicated due to more complicated structure of its time-Lax operator $V^{(k)}$. Since we have presented in detail the DNLSE case given by $k=2$, we report here in brief the construction from the next equation (5.13) obtained for $k=3$, which yields

$$
\begin{aligned}
Q_{t_{3}} & -Q_{x x x}+3 i \sigma\left(|Q|^{2} Q_{x}\right)_{x}+\frac{3}{2} \sigma^{2}\left(\left(|Q|^{4} Q\right)_{x}+i \theta_{x}^{(n)}|Q|^{4} Q\right) \\
& -3 i \theta_{x}^{(n)} Q_{x x}-3\left(i \theta_{x x}^{(n)}-\left(\theta_{x}^{(n)}\right)^{2}\right) Q_{x}-3 \sigma\left(\left(|Q|^{2} \theta_{x}^{(n)} Q\right)_{x}+\theta_{x}^{(n)}|Q|^{2}\left(Q_{x}-i \theta_{x}^{(n)} Q\right)\right) \\
& +\left(i \theta_{t_{3}}^{(n)}+3 \theta_{x}^{(n)} \theta_{x x}^{(n)}-i \theta_{x x x}^{(n)}+i\left(\theta_{x}^{(n)}\right)^{3}\right) Q=0 .
\end{aligned}
$$

By inserting $\theta_{x}^{(n)}$ from (5.7) and deriving the consistent $\theta_{t_{3}}^{(n)}$ we can generate from (5.14) the EK type integrable hierarchy with $n=0,1, \ldots$, preserving its 3rd-order LD.

The simplest and the lowest equation of this hierarchy with $n=0$ may be given explicitly by the choice of the gauge function:

$$
\begin{aligned}
\theta_{x}^{(0)}= & \delta|Q|^{2} \\
\theta_{t_{3}}^{(0)}= & \delta\left(Q^{*} Q_{x x}+Q Q_{x x}^{*}-Q_{x}^{*} Q_{x}+3 i(\alpha+\delta)|Q|^{2}\left(Q^{*} Q_{x}-Q Q_{x}^{*}\right)\right. \\
& \left.-3\left(2 \delta \alpha+\delta^{2}-\frac{1}{2} \alpha^{2}\right)|Q|^{6}\right),
\end{aligned}
$$

consistency of which can be checked by using the originating equation (5.13). Inserting (5.15) in (5.14) one would clearly get an integrable equation having nonlinearity up to 7 -th order in the field including its derivatives, but with the linear dispersive term $Q_{x x x}$ as in (5.13). 


\section{Concluding remarks}

Our construction of new EK type hierarchies of integrable equations with higher and higher nonlinearities, extending the NLSE and the DNLSE demonstrates clearly that suitable combinations of nonlinear terms may be added to the original integrable equations without changing their linear dispersion relation, and at the same time preserving their integrability.

Moreover, one can apply the whole procedure to each of the $k=3,4, \ldots$ equations in the known integrable NLSE and DNLSE hierarchies, containing $k$ th order linear dispersion. Each of such equations therefore would generate its new integrable EK hierarchy with higher nonlinear terms, but with the same linear dispersive term.

It may be recalled that the generalized higher NLSE appearing in many physical problems [2, $3,4,5,6,7]$, which contains higher nonlinear terms together with the same second-order linear dispersive term, can be reduced for particular parameter choices to integrable equations (2.3) or (2.4). Therefore we may hope that equations with hopelessly complicated nonlinear terms, arising in many other physical situations, could also be reducible to some of the higher nonlinear equations in the rich integrable EK hierarchies found here and hence could be exactly solved, at least for certain parameter choice or for some limiting values. The method presented here for constructing new integrable nonlinear hierarchies with unchanged linear dispersion is general enough to be applicable to other integrable PDEs with complex fields, e.g. mixed DNLS, complex $\mathrm{mKdV}$ etc. and even to vector models like Manakov model.

[1] Agarwal G., Nonlinear fiber optics, Elsevier, 2001, p. 50.

[2] Johnson R.S., On the modulation of water waves in the neighbourhood of $k h \approx 1.363$, Proc. Roy. Soc. London Ser. A, 1977, V.357, 131-141.

[3] Benney D.J., A general theory for interactions between short and long waves, Studies in Appl. Math., 1976/77, V.57, 81-94.

[4] Kakutani T., Michihiro K., Marginal state of modulational instability-mode of Benjamin Feir instability, J. Phys. Soc. Japan, 1983, V.52, 4129-4137.

[5] Parkes E.J., The modulation of weakly non-linear dispersive waves near the marginal state of instability, J. Phys. A: Math. Gen., 1987, V.20, 2025-2036.

[6] Ndohi R., Kofane T.C., Solitary waves in ferromagnetic chains near the marginal state of instabilit, Phys. Lett. A, 1991, V.154, 377-380.

[7] Pelap F.B., Faye M.M., Solitonlike excitations in a one-dimensional electrical transmission line, J. Math. Phys., 2005, V.46, 033502, 10 pages.

[8] Sakovich S.Yu., Integrability of the higher order NLS revisited, nlin.SI/9906012.

[9] Kindyak A.S., Scott M.M., Patton C.E., Theoretical analysis of nonlinear pulse propagation in ferritedielectric-metal structures based on the nonlinear Schrödinger equation with higher order terms, J. Appl. Phys., 2003, V.93, 4739-4745.

[10] Zarmi Y., Perturbed NLS and asymptotic integrability, nlin.SI/0511057.

[11] Kundu A., Landau-Lifshitz and higher-order nonlinear systems gauge generated from nonlinear Schrödingertype equations, J. Math. Phys., 1984, V.25, 3433-3438.

[12] Calogero F., Eckhaus W., Nonlinear evolution equations, rescalings, model PDEs and their integrability. I, Inverse Problems, 1987, V.3, 229-262.

[13] Clarkson P.A., Cosgrove C.M., Painlevé analysis of the nonlinear Schrödinger family of equations, J. Phys. A: Math. Gen., 1987, V.20, 2003-2024.

[14] Kakei S., Sasa N. Satsuma J., Bilinearization of a generalized derivative nonlinear Schrödinger equation, J. Phys. Soc. Japan, 1995, V.64, 1519-1523, solv-int/9501005.

[15] Feng Z., Wang X., Explicit exact solitary wave solutions for the Kundu equation and the derivative Schrödinger equation, Phys. Scripta, 2001, V.64, 7-14.

[16] Shen L.Y., Some algebraic properties of c-integrable nonlinear equation II-Eckhaus-Kundu equation and Thomas equation, Preprint, Univ. Sc. Tech. China, Hefei, China, 1989. 
[17] Shen L.Y., Symmetries and constants of motion of integrable systems, in Symmetries \& Singularity Structures, Spinger, 1990, 27-41.

[18] Conte R., Musette M., The Painlevé methods, in Classical and Quantum Nonlinear Integrable Systems, Bristol, IOP Publ., 2003, 39-63.

[19] Chen H.H., Lee Y.C., Liu C.S., Integrability of nonlinear Hamiltonian systems by inverse scattering method, Phys. Scripta, 1979, V.20, 490-492.

[20] Gerdjikov V.S., Ivanov M.I., The quadratic bundle of general form and the nonlinear evolution equations: hierarchies of Hamiltonian structures, JINP Preprint E2-82-595, Dubna, 1982, 16 pages.

[21] Mikhailov A.V., Shabat A.B., Yamilov R.I., Extension of the module of invertible transformations. Classification of integrable systems, Comm. Math. Phys., 1988, V.115, 1-19.

[22] Mikhailov A.V., Shabat A.B., Yamilov R.I., A symmetric approach to the classification of nonlinear equations. Complete lists of integrable systems, Uspekhi Mat. Nauk, 1987, V.42, 3-53.

[23] Ablowitz M., Segur H., Solitons and the inverse scattering transform, Philadelphia, SIAM, 1981 , p. 54.

[24] Novikov S.P. (Editor), Theory of solitons, Moscow, Nauka, 1980, p. 76 (in Russian).

[25] Kaup D.J., Newell A.C., An exact solution for a derivative nonlinear Schrödinger equation, J. Math. Phys., 1978, V.19, 798-801. 